\title{
NUEVAS FÓRMULAS EN TORNO A LA RESPONSABILIDAD DE LOS SOCIOS DE LAS COOPERATIVAS EN EUSKADI
}

\author{
Miren Epelde Juaristi \\ Doctoranda \\ Departamento de Derecho de la Empresa \\ Universidad del País Vasco/Euskal Herriko Unibertsitatea (UPV/EHU) \\ https://orcid.org/0000-0002-7993-0467
}

\section{RESUMEN}

Es patente la apuesta de la Unión Europea por un mercado con mayor presencia de las empresas de la economía social y consideración hacia las personas trabajadoras, como es el caso de la sociedad cooperativa. A pesar de su tradición, en su regulación siguen existiendo apartados que pueden ser interpretados de forma diversa o que generan dudas, como los relativos a la responsabilidad de los socios. Si bien se deduce que la responsabilidad por deudas sociales es claramente limitada, surgen dudas en torno a la imputación de pérdidas. Tras años de investigación y negociación con los agentes implicados, en diciembre de 2019 se aprobó la nueva Ley de cooperativas de Euskadi, donde el legislador opta por aclarar que la responsabilidad de los socios por las deudas sociales es limitada y establece fórmulas novedosas para la imputación de las perdidas, sin menoscabo al patrimonio personal de los socios cooperativistas.

PALABRAS CLAVE: Sociedad Cooperativa, responsabilidad del socio, deudas sociales, perdidas, baja del socio.

CLAVES ECONLIT / ECONLIT DESCRIPTORS: P13, J54, C71.

Cómo citar este artículo/How to cite this article: EPELDE JUARISTI, M.: "Nuevas fórmulas en torno a la responsabilidad de los socios de las cooperativas en Euskadi", CIRIEC-España, Revista Jurídica de Economía Social y Cooperativa, n³ 37, 2020, pp. 141-165. D0I: 10.7203/ CIRIEC-JUR.37.17752. 


\section{NEW TOOLS FOR THE LIABILITY OF THE COOPERATIVE PARTNERS IN THE BASQUE COUNTRY}

\section{EXPANDED ABSTRACT}

It goes without saying that the world is involved in a global economic crisis. As we know, the Covid-19 is the main reason of this situation. As a result, the industry and in general the economy is making a big effort to survive. However, the last economic crisis the world suffered did not happen a long time ago. The measures adopted in that crisis were not the best ones for the economy, although, one thing was proven, the social economy would be the key to get out of the crisis.

Until now the economic system was focused on two aspects: capitalism economy and public economy. However, the third pillar, which is the social economy, was not considered. Nowadays the experts are realizing that the economic system used until now was not efficient and a change was needed.

The social economy takes into account the employees and it is more focused on the personal wellness. Due to that fact, the motivation of the employees often increases. They would feel like they are part of something and because of that if the workers are happy in their daily routine and they know that if they do their best, the company will answer in a good sense for their personal life, they would work better. As a result, the company's productivity would be higher and good economic results would provide for the survival of the company and also a job for workers.

There is a well-known entity in the world which strongly considers the social economy. They are called cooperatives and because of their way to organize are considered part of the social economy. In the last decade cooperatives have been the key to survival for many companies in the European and Spanish market. The crisis that started in 2007 and the following ones greatly affected many factories, and as a result their futures were uncertain. Many factories turned to social economics as their solution.

It seems, though, that as time goes by the shadow of the crisis still looms. As a result, many leaders have based their political agendas on the necessity of contributing to the economic transformation in order to survive and adapt to the current marketplace. To do so, they intend on using new business practices and economic agents. In this way the European Union has placed a big bet on social economics, and has given more consideration to employees as is the case in a cooperative society. When the economic crisis concerns to different Eu- 
ropean countries, Union's help has to be strong and effective to give advice and try to find a Union solution.

However, the legal ambiguity that exists in this area allows for the possibility of making different interpretations about the same regulation. Such is the case when it comes to assessing the liability of a cooperative member. Taking the Basque and Spanish regulations into account, it is concluded that a partner's liability in regard to social debts is limited to the contribution made by them. Nevertheless, there is some doubt about how limited the liability of a partner is when a factory has losses and they can be attributed to them.

The State Cooperatives law is the $27 / 1999$, of $16^{\text {th }}$ of July. According to this law the liability of the partner's in the case of debts is limited to the input made by them. Nevertheless, the answer when there are losses is not the same. The law allows the possibility of attributing the losses to the partners. Something similar happens in the Basque Country where there are many cooperatives.

The law to regulate cooperatives in the Basque Country was the $4 / 1993$, of $24^{\text {th }}$ of June. According to this regulation, in the case that a factory has losses, there is a possibility of attributing them to the partners. This fact wasn't good for the partners. After the last crisis suffered in 2007, employee saw that the attribution of losses to them was real, and due to that, their personal assets would be affected. As a result, they asked for a law change.

After years of research and negotiations with representatives from different cooperatives, the Basque government has enacted a new law in order to clarify the ambiguities present in the 1993 law. The new regulation states that partner's liability when it comes to social debt continues to be limited. In the case of the responsibility created by yearly losses, the new 2019 law approves new ways for a partner to be liable without affecting their personal assets. It says that first of all the losses have to be covered by the voluntary reserves. However, if they are not enough, obligatory reserves would be affected. And as the last chance there is a possibility to attribute them to the partner's.

Another point to take into account is the option of creating a special account, and cover the losses with the surplus of the next years. These two ways are optional, because is deducing from the law that they are optional, and so that it is the option of choosing one or another way. With this interpretation, it seems that the partners are not going to respond with their own personal assets. Nevertheless, the innovation of the law appears in the last point, where it is said that capital increase would be possible to face the losses if previous measures are not enough and as a result not undermine the partner's personal assets. 
Moreover, the situation of partner's termination or their decision to leave the cooperative also creates ambiguity in the interpretation, due to the fact that the state regulation and Basque Country regulation is not the same. The state law says that partners are liable of the debts generated in the cooperative during five years since their termination. However, the Basque legislator says that there is not option to be liable after leaving the company. With the new regulation, some of this aspects were changed, and there is an option to be liable of the decisions which were taken when the partner was member of the cooperative.

Therefore, the goal of this article is to clarify the aforementioned legal ambiguity, and in order to do that, the new regulation in going to be analyzed.

KEYWORDS: Cooperative society, partner's liability, social debts, losses and partner's termination. 


\section{SUMARIO}

1. Introducción. 2. Ambigüedad en la normativa básica. 2.1. Responsabilidad por deudas. 2.2. Responsabilidad por pérdidas. 3. Nuevas fórmulas en la Ley de Cooperativas de Euskadi. 3.1. Régimen de imputación de pérdidas. 3.2. Baja del socio y deudas de la sociedad. 4. Conclusiones. Bibliografía.

\section{Introducción}

Es tiempo de incertidumbre para la economía debido a la crisis generada por el COVID 19, una situación que no se esperaba y que ha repercutido en todo el mundo sin excepción. Es por ello, que la economía debe de amoldarse a las nuevas situaciones y emprender un camino renovado con capacidad de cambio y adaptabilidad a la nueva era.

Actualmente la tasa de paro en España es del 14,41\% y, en el caso concreto de Euskadi, se sitúa en el 8,03\% en las personas empleadas mayores de 25 años ${ }^{1}$. Como ocurrió en la crisis pasada, los datos actuales de desempleo aumentarán cada día, ya que, los graves problemas sanitarios están generando una gran crisis económica. Las medidas del gobierno hasta ahora adoptadas han ayudado a mantener cierta estabilidad en el empleo, pero es y será inevitable el incremento del desempleo ante una situación de tal magnitud. Es por ello que las medidas económicas que se tomarán en el futuro deben de responder a la situación real del mercado. Se dice que la economía social puede jugar un gran papel en la "nueva realidad", puesto que ha dado muestras de que en tiempos de crisis puede ayudar a sostener y mantener los empleos y las empresas. Es decir, contribuye a mantener en cierta medida el tejido empresarial en cada territorio y, en opinión de algunos, constituye incluso una salida a la crisis económica ${ }^{2}$.

1. Instituto Nacional de Estadística, consultado el 07/05/2020. En:

https://www.ine.es/jaxiT3/Datos.htm?t=4247\#!tabs-tabla

2. Como afirma Juan Antonio Pedreño, presidente del Confederación Empresarial Española de Economía Social, (CEPES): "las empresas resilientes, que anteponen las personas y el objeto social a los beneficios y que vamos a ser con toda seguridad un agente clave en la recuperación económica y social que España necesita", consultado el 07/05/2020. En:

http://www.observatorioeconomiasocial.es/actualidad-observatorio.php?id=4518\&PHPSESSID=-

94498b290af8de0e3c00a9e928dbb04d 
En ese contexto se sitúan las cooperativas, que se han integrado cada vez más en el tejido empresarial de nuestro país. En el caso de Euskadi, esta figura lleva años formando parte del catálogo de posibles formas jurídicas de las empresas, teniendo en cuenta que la Corporación Cooperativa Mondragón ha servido de ejemplo para decenas de empresas cooperativas creadas en el territorio. Esta Corporación sufrió su gran expansión en los años sesenta en la zona de Arrasate (Guipuzcoa), y han sido capaces de mantener esa expansión durante las siguientes décadas, con la creación de nuevas cooperativas ${ }^{3}$.

Sin embargo, a nadie se le escapa que la última crisis económica de 2007 azotó a sectores de todo tipo, tal y como ocurre en la mayoría de las crisis económicas, incluyendo así a las cooperativas. La empresa Fagor Electrodomésticos fue la más afectada y renombrada en el caso de Euskadi. Las ventas de la empresa cayeron un 37\% y la plantilla sufrió una reducción del 50\%, generando así una deuda de 700-800 millones de euros. Las pérdidas llegaron a ascender a 60,4 millones de euros ${ }^{4}$, siendo inviable la sostenibilidad de la empresa y declarándose el concurso de la misma. Este concurso conllevó a los trabajadores la obligación de hacer frente a las pérdidas con su propio patrimonio personal, puesto que así lo dictamino el juez. Según la sentencia había prescrito el plazo para interponer la acción de reclamación de la responsabilidad extracontractual de $\mathrm{MCC}^{5}$. La sentencia en cuestión supuso un gran revés para el conjunto de los trabajadores de las cooperativas, ya que, generaba jurisprudencia y, por ende, la consiguiente inseguridad y preocupación para todos ellos.

En cuanto al marco normativo, la Ley estatal de cooperativas sirve de punto de referencia básico de esta forma social, si bien prácticamente todas las Comunidades Autónomas (salvo Ceuta y Melilla) han asumido la competencia exclusiva en esta materia, por lo que existe una pluralidad de leyes autonómicas al respecto. Sin embargo, tal y como ocurre con otras muchas leyes, puede decirse que se aprecia cierto vacío legal en el ámbito de la limitación de la responsabilidad de los socios.

3. Vid. BAKAIKOA AZURMENDI B.: "El cooperativismo vasco. El ejemplo de la Mondragon Corporación Cooperativa". En: El cooperativismo Vasco y el año 2000, Marcial Pons, Ediciones Jurídicas y Sociales \& Gezki, Madrid, 1995, pp. 29-47. Asimismo, vid. BAKAIKOA AZURMENDI, B., ETXEZARRETA ETXARRI, E. \& MORANDEIRA ARCA, J.: "Regeneración de empresas de la economía social: un reto en el País Vasco", REVESCO. Revista de Estudios Cooperativos, nº. 112, noviembre, 2013, pp. 151-175.

4. Noticia de EITB, consultado el 07/05/2020. En: http://www.eitb.com/eu/albisteak/ekonomia/osoa/1675780/fagor-etxetresnak--kooperatibaren-krisiazenbakitan/

5. UPAD del Juzgado de Primera Instancia e Instrucción no 1 de Bergara, Sentencia nº. 70/2018, de 27 de julio, (Procedimiento ordinario 204/2017-C). 
En el caso de las empresas de capital, la responsabilidad de cada socio se encuentra bien delimitada sin problemas de interpretación, pero en el ámbito de las cooperativas la situación es bien distinta. Sin embargo, en el ámbito de las cooperativas, aunque en teoría parezca que la responsabilidad es limitada, se ha visto en la práctica que la normativa autonómica (y también la estatal) apelaba a diferentes interpretaciones que podrían ser perniciosas para los trabajadores.

Ante esta situación y dado el crecimiento de las cooperativas en Euskadi, del $30,05 \%$ entre los años $2004-2014^{6}$, alcanzando la cifra total de 57.815 cooperativas en el año 20167 , el Gobierno Vasco, junto con la Federación de Cooperativas de Trabajo Asociado, Enseñanza, Consumo y Crédito de Euskadi (en adelante ERKIDE) después de haber estudiado e intercambiado opiniones con expertos e interesados, estimó oportuno emprender la renovación de la ley para aclarar ciertos aspectos de la misma que generaban dudas a la hora de su aplicación práctica. La Ley 11/2019, de 20 de diciembre, de Cooperativas de Euskadi (BOPV no 247) vino a introducir algunos aspectos novedosos en torno a la responsabilidad de los socios por perdidas de la cooperativa a cuyo estudio está dedicado el presente trabajo.

\section{Ambigüedad en la normativa básica}

En materia de cooperativas, cada comunidad autónoma cuenta con capacidad para la elaboración de su propia normativa, atendiendo a las circunstancias del tejido empresarial de la zona. Ante la diversidad de regulaciones, la aparición de ambigüedades normativas es un hecho casi inevitable ${ }^{8}$. A ello hay que añadir que la mayoría de regulaciones sobre cooperativas no resultan ser muy actuales, dado que la normativa en general está basada en la economía de los años noventa. Todos estos factores influyen en el tema que nos ocupa de la responsabilidad de los socios. A este respecto, se impone realizar una diferenciación básica entre el concepto de deudas y cómo responde el socio ante ellas y la figura de las pérdidas y su repercusión en el patrimonio personal de los socios.

6. Vid. ORTEGA I.: Aportación de las Cooperativas al resto del tejido vasco. Mondragon Unibertsitatea, Mondragón, 2018.

7. Vid. SOTO GORROTXATEGI, A. \& MORANDEIRA ARCA, J.: Informe de situación de la Economía Social Vasca año 2016, Observatorio Vasco de Economía Social / Gizarte Ekonomiako Euskal Behatokia (OVES/GEEB) \& Gezki, Donostia, 2016.

8. Al respecto, vid. entre otros, la visión crítica de: EMBID IRUJO, J.M.: "El relieve del Derecho de sociedades para la ordenación jurídica de las empresas de economía social”, Cuadernos de Derecho y Comercio, n 71 , 2019, p. 31; que califica esta pluralidad de regulaciones de injustificada e inconveniente. 


\subsection{Responsabilidad por deudas}

Obviamente, el funcionamiento de la sociedad cooperativa trae consigo la generación de responsabilidades con terceros que, en caso de una mala gestión administrativa, o una crisis económica puede conducir a la generación de deudas. Se entiende por deuda toda obligación adquirida por la sociedad que no ha sido pagada. Se deduce de la interpretación de la Ley 27/1999, de 16 de julio, de Cooperativas estatal, (en adelante, LCoop) que es la sociedad cooperativa la responsable frente a esas deudas. Esto es, es la persona jurídica la que con su patrimonio tiene que hacer frente a esas obligaciones contraídas por ella.

El patrimonio de la sociedad cooperativa está constituido por las aportaciones realizadas por los socios. Éstos se comprometen a realizar una serie de aportaciones sociales, limitando con ello el umbral de su responsabilidad ${ }^{9}$. Ello incluye tanto las aportaciones al capital social como las demás aportaciones voluntarias u obligatorias. La exigencia de estas últimas aportaciones se recogerá en los estatutos de la empresa y serán necesarios para la creación de fondos de reserva. Estos fondos suelen estar destinados a la supervivencia de la sociedad, juegan un papel de garantía de la mis$\mathrm{ma}$, siendo unos fondos inembargables ${ }^{10} \mathrm{e}$ irrepartibles ${ }^{11}$. Sin embargo, la aportación realizada al capital social es la que responde ante las deudas generadas. En este apartado, a pesar de la pluralidad normativa por parte de las comunidades autónomas, las diferentes leyes coinciden y su interpretación es la misma. La regla es que los socios responden ante las deudas generadas de forma limitada, ciñéndose a la aportación realizada al capital social.

Con todo, también es posible avistar un escenario donde la responsabilidad del socio puede ser ilimitada, teniendo que responder con todos sus bienes presentes y

9. LCoop, artículo 15.3: "La responsabilidad del socio por las deudas sociales estará limitada a las aportaciones al capital social que hubiera suscrito, estén o no desembolsadas en su totalidad”.

10. Vid. BELTRÁN SÁNCHEZ, E.M.: "Reforma de la Ley de cooperativas". En: Comentario de la Ley Concursal (dirs. ROJO FERNÁNDEZ-RÍO, A., BELTRÁN SÁNCHEZ, E.M. \& coord. CAMPUZANO LAGUILLO, A.B.), Civitas, Madrid, 2006, pp. 3267-3268. Asimismo, vid. GRIMALDOS GARCÍA, M.I.: "El concurso de la cooperativa y su repercusión en el patrimonio personal de los socios", Revista de Derecho Concursal y Paraconcursal, no 11, 2009, pp. 323-343.

11. In extenso, sobre los fondos irrepartibles de las cooperativas, vid. la tesis doctoral ALZOLA BERRIOZABALGOITIA, M.I.: El régimen jurídico de los fondos de reserva de las sociedades cooperativas [Tesis doctoral, Mondragon Unibertsitatea], Mondragón, 2009; y: ALZOLA BERRIOZABALGOITIA, M.I. \& VILLAFÁNEZZ PÉREZ, I.: "Los recursos financieros en la legislación cooperativa y sus peculiaridades en las cooperativas de trabajo asociado". En: Cooperativa de trabajo asociado y estatuto jurídico de sus socios trabajadores (dir. FAJARDO GARCÍA, I.G. \& coord. SENENT VIDAL, M.J.), Tirant lo Blanch, València, 2016, pp. 481-512. Asimismo, vid. VITERI ZUBIA, I.: "La responsabilidad del socio cooperativista por las pérdidas sociales", CIRIEC-España, Revista Jurídica de Economía Social y Cooperativa, no 28, julio 2016, p. 214. 
futuros, en caso de pérdidas de la sociedad. En estos casos, la responsabilidad de los socios prevista estatutarimente suele ser mancomunada, de forma que se divide entre todos y cada uno de ellos para que hagan frente a su parte alícuota de responsabilidad de forma personal e ilimitada, con todos su bienes presentes y futuros (art. 1911 CC). Pero también cabe la posibilidad de decidir mediante cláusula estatutaria que la responsabilidad sea solidaria entre la cooperativa y los socios, dando la opción a los acreedores de poder reclamar la deuda a quien estime oportuno ${ }^{12}$.

Además de estos dos posibles tipos de responsabilidad de los socios vía estatutos, cabe añadir una tercera opción que sería la de una responsabilidad adicional. En este caso, en principio, el socio respondería de forma limitada por las deudas sociales, esto es, respondería únicamente con la parte aportada al capital de la empresa. Pero podría matizarse en los estatutos que, para determinadas situaciones de extrema necesidad, como puede ser, por ejemplo, la de insolvencia, el socio deba responder ilimitadamente.

Por lo tanto, en el ámbito de las deudas generadas por la sociedad, queda claro que la aportación realizada al capital social es la que responde ante las deudas generadas. Esa es la regla general y, en este apartado las legislaciones autonómicas son coincidentes.

\subsection{Responsabilidad por pérdidas}

Sin embargo, no ocurre así en el ámbito de las pérdidas, que tienen un significado distinto. La pérdida es una disminución del activo de la empresa, es decir, una minoración de los bienes y derechos. Es un concepto contable para referirse a situaciones con resultados negativos. Esos resultados surgen al cierre de un ejercicio económico cuando los gastos son superiores a los ingresos (vg. cuando la empresa no ha vendido todo lo producido durante el año) generando un saldo negativo. Para estas situaciones, el tratamiento otorgado por la legislación a la responsabilidad de los socios es diferente.

El articulo 15 LCoop entra en juego con el 59 LCoop, lo que da pie a la posibilidad de imputar las pérdidas a los socios. Según el artículo 15.3 LCoop, está claro que la responsabilidad del socio queda limitada a la aportación realizada a la sociedad. Esto es, el socio responde limitadamente ante las deudas sociales. Pero la duda se plantea con el texto del artículo 59 LCoop.

12. Vid. ARCO ÁlVAREZ, J.L.: Teoría y práctica de las sociedades cooperativas. Tema de estudio de los Curso I, II y III, Universidad Politècnica de València (UPV), València, 1974, p. 55. 
En su primer apartado se dispone que, los estatutos pueden prever la posibilidad de imputar las pérdidas a una cuenta especial para su amortización con cargo a futuros resultados positivos, dentro del plazo máximo de siete ańos, fijando los criterios para ello.

El segundo apartado, establece las reglas a las que deberá sujetarse la cooperativa para la compensación de las pérdidas. (a) Podrán imputarse, en su totalidad, a los fondos de reserva voluntarios, si existiesen. En este caso, es obvio que no se imputa la pérdida directamente al socio, sino a la sociedad. (b) También podrán imputarse al fondo de reserva obligatorio, pero con limitaciones ${ }^{13}$. En este caso se sigue respetando la responsabilidad limitada del socio. (c) Pero finalmente prevé la imputación de las pérdidas al socio, vinculando así su patrimonio privado, cuando las dos opciones anteriores no sean suficientes ${ }^{14}$. Por lo tanto, con ello se pone en tela de juicio la responsabilidad limitada de los socios que predica el artículo 15.3 LCoop.

Así, sobre la base de nuestra premisa, en línea con la distinción de la que partimos, la lectura del artículo 59 LCoop nos lleva a deducir que el socio es responsable limitadamente de las deudas generadas por la sociedad, pero no, de las pérdidas. Ésa parece ser la teleología de la norma.

Además, el precepto regula diferentes modalidades para que los socios satisfagan las pérdidas, lo cual induce a pensar en el sentido indicado. Por un lado, se podría determinar que el socio es responsable de las pérdidas generadas, imputándolas a la aportación realizada al capital social o a las inversiones en la cooperativa. Otra opción posible sería la de imputar los posibles retornos que pueda sufrir el socio durante los siguientes siete años. Una vez transcurrido este plazo y si la pérdida estuviera todavía sin compensar, el socio gozaría de un mes de gracia para realizar el pago.

Sin embargo, la doctrina se encuentra dividida sobre este tema, con argumentos de un lado y de otro. En opinión de algunos, el socio nunca deberá de ser responsable más allá de la aportación realizada ${ }^{15}$, otros, al contrario, entienden que sí lo puede

13. LCoop artículo 59.2.b): "Al fondo de reserva obligatorio podrán imputarse, como máximo, dependiendo del origen de las pérdidas, los porcentajes medios de los excedentes cooperativos o beneficios extracooperativos y extraordinarios que se hayan destinado a dicho fondo en los últimos cinco años o desde su constitución, si ésta no fuera anterior a dichos cinco años".

14. LCoop artículo 59.2.c): "La cuantía no compensada con los fondos obligatorios y voluntarios se imputará a los socios en proporción a las operaciones, servicios o actividades realizadas por cada uno de ellos con la cooperativa. Si estas operaciones o servicios realizados fueran inferiores a los que como mínimo está obligado a realizar el socio conforme a lo establecido en el artículo 15.2.b), la imputación de las referidas pérdidas se efectuará en proporción a la actividad cooperativizada mínima obligatoria”.

15. En este sentido, vid. MATEO BLANCO, J.: El retorno cooperativo, Caja Rural de Zaragoza, Zaragoza, 2003; PAZ CANALEJO, N.: El nuevo derecho cooperativo español, Digesa, Madrid, 1979, p. 72; VICENT 
$\operatorname{ser}^{16}$. Con todo, la responsabilidad limitada de los socios no parece evidente y corre el riesgo de quedar trastocada, condicionada a la imputación de pérdidas.

\section{Nuevas fórmulas en la Ley de Cooperativas de Euskadi}

En la Comunidad Autónoma del País Vasco se repetía la misma incertidumbre en torno a la responsabilidad de los socios. La normativa autonómica ${ }^{17}$ también regulaba esa posibilidad de imputar pérdidas a los socios, más allá de la aportación realizada al capital social, y ello generaba dudas sobre el alcance real de su responsabilidad.

Cuando la crisis de 2007 golpeó de lleno a la economía, las cooperativas no fueron una excepción. Esta situación sacó a la luz el hecho de que los socios cooperativistas no gozaban de plena garantía de protección de su patrimonio personal por la actividad de la sociedad, lo que fue generando una movilización de acercamiento a las estructuras de las sociedades capitalistas. Los socios trabajadores reclamaban una mayor seguridad y protección en torno al alcance de su potencial responsabilidad por las deudas y pérdidas de la empresa, a semejanza de los socios de las sociedades de capital ${ }^{18}$.

En el País Vasco, la norma vigente hasta finales de 2019 fue la Ley $4 / 1993^{19}$ de Cooperativas de Euskadi (en adelante, Ley 4/1993), aunque acompañada de nume-

CHULIÁ, F.: Ley general de cooperativas, Tomo XX de los Comentarios al Código de comercio y Legislación mercantil Especial, vol. 3, Madrid, 1994, pp. 372 y ss; ibid.: Introducción al derecho mercantil, vol. 1, Tirant lo Blanch, València, 2012, pp. 1186-1191; y: FAJARDO GARCÍA, I.G.: "La gestión económica de las cooperativas: responsabilidad de los socios". En: Confederación de cooperativas de la comunidad Valenciana, Capitulo V, Editorial Tecnos, Madrid, 1997, pp. 197-245.

16. Al respecto, vid. ELENA DIAZ, F.: "Ideas sobre una posible interpretación de las pérdidas del pasivo según la vigente ley”, Estudios cooperativos, no 36-38, 1975-1976, pp. 190; LARRAÑAGA, J.: Análisis de la Legislación Vasca sobre cooperativas, Caja Laboral Popular, Mondragón, 1985, p. 34; VILLA GÓMEZ, A.: Comentarios a la ley de cooperativas 27/1999 de 16 de julio, vol. I, Madrid, 2001, pp. 294 y ss; y: GADEA SOLER, E., SACRISTÁN BERGIA, F. \& VARGAS VASSEROT, C.: Régimen jurídico de la sociedad cooperativa del siglo XXI. Realidad actual y propuestas de reforma, Dykinson, Madrid, 2009.

17. En caso de la Comunidad Autónoma Vasca, se recoge en su Estatuto de Autonomía en el artículo 10.23 la competencia exclusiva en la materia, quedando así justificada la competencia de regulación propia.

18. Vid. IRASTORZA MARTÍNEZ, I. \& LÓPEZ LOLO, R.: "Acercamiento del régimen de las sociedades cooperativas al de las sociedades de capital. ¿Un cambio necesario para el futuro del modelo cooperativo?”, Diario la Ley, no 9582, 2020.

19. Ley 4/1993 de Cooperativas de Euskadi, BOPV nº. 135, de 24 de junio de 1993. 
rosas normas y reformas sucesivas ${ }^{20}$, siendo referente para muchas Comunidades Autónomas, por ser una de las primeras en asumir la competencia exclusiva en materia de cooperativas junto con Cataluña, seguidas de Andalucía, Valencia y Navarra ${ }^{21}$. Los cambios normativos en Euskadi desde su primera Ley de Cooperativas de 1982 no han sido pocos. La práctica del día a día saca a relucir las debilidades de las leyes por lo que es deber del legislador atender y dar respuesta a esas necesidades, ya que, el legislador vasco intenta adecuarse a la situación real del mercado en cada momento. Así, en 2019, tras años de análisis y entendiendo que existía la necesidad de aclarar ciertos aspectos de la Ley 4/1993, se aprobó la nueva Ley de Cooperativas de Euskadi ${ }^{22}$ (en adelante, Ley 11/2019).

Debe reconocerse que este último cambio ha sido promovido, en gran parte, por la crisis de 2007 a raíz de la cual, entre otras cuestiones, se agudizaron las dudas sobre cómo debía de interpretarse la normativa sobre la responsabilidad de los socios, puesto que no se sabía a ciencia cierta hasta dónde llegaba. Como se ha comentado, la ambigüedad de las leyes (tanto estatal como vasca) daba pie a diferentes interpretaciones por parte de los jueces, no teniendo así un criterio claro para resolver los problemas planteados. ¿¿Hasta dónde se le podía exigir responsabilidades al socio por la imputación de pérdidas de la cooperativa? Éstas y otras cuestiones pesaron mucho en la sociedad vasca, con una economía con fuerte presencia de las cooperativas, por lo que se hizo evidente la imperiosa necesidad de un cambio normativo.

Un claro exponente de los riesgos derivados de la ambigüedad a la que nos hemos venido refiriendo fueron las sentencias dictadas en el caso de la cooperativa Fagor

20. Vid. el Reglamento sobre Procedimientos de resolución en Conflictos en las Cooperativas Vascas; Real Decreto 64/1999, de 2 de febrero, por el que se aprueba el Reglamento sobre procedimientos y requisitos relativos a las sociedades cooperativas de utilidad pública; Real Decreto 61/2000, de 4 de abril, por el que se regulan las Cooperativas de Iniciativa Social; Real Decreto 59/2005, de 29 de marzo, por el que se aprueba el Reglamento de Organización y Funcionamiento del Registro de Cooperativas de Euskadi; Ley 27/1999, de 16 de julio, de Cooperativas; Ley 4/1993, de 24 de junio, de Cooperativas de Euskadi. Esta última ha sufrido varias modificaciones en virtud de la Ley 1/2000, de 29 de junio, de modificación de la Ley de Cooperativas de Euskadi, Ley 8/2006, de 1 de diciembre, de segunda modificación de la Ley de Cooperativas de Euskadi y disposición adicional cuarta a la Ley 6/2008, de 25 de junio, de la Sociedad Cooperativa Pequeńa de Euskadi, por la que se modifican los artículos 67 y 68 de la Ley 4/1993.

21. A la ley de 1993 le precedió la Ley de cooperativas de Euskadi de 11 de febrero de 1982. Vid. ATIENZA MAZIAS, E., MERINO MAR, J.A. \& GUILLERMO RUIZ DE HUYDOBRO Y DE LAS MUÑECAS, E.: "Derecho de las Sociedades Cooperativas en Euskadi: Un análisis sobre su estructura orgánica y política cooperativa de los últimos ejercicios", Boletín de la Asociación Internacional de Derecho Cooperativo (BAIDC), no 38, 2004, pp. 111 y ss.

22. Ley 11/2019 de Cooperativas de Euskadi, BOPV nº. 247, de 30 de diciembre de 2019. 
Electrodomésticos del grupo Mondragón ${ }^{23}$, que se vio envuelta en una situación de insolvencia muy grave, en la que quedó totalmente desprotegido el patrimonio personal de los socios trabajadores ${ }^{24}$. La sentencia de la Audiencia Provincial vino a ratificar lo dictado por el juez de Primera Instancia de Bergara, entendiendo que no se cumplió con el plazo para presentar la acción de reclamación extracontractual de MCC. Ante una situación de insolvencia de la cooperativa, las pérdidas pagaderas que no se pudieron cubrir con el patrimonio de la sociedad fueron imputadas a los socios, disponiendo así los acreedores de la opción de acceder al patrimonio personal de cada uno de ellos.

Esta situación y la consiguiente decisión judicial hacían tambalear la estabilidad de las cooperativas en Euskadi, ya que, dejaba totalmente desprotegidos a los trabajadores. Fueron ańos oscuros, especialmente para los socios trabajadores de Fagor Electrodomésticos ${ }^{25}$, por lo que se hizo absolutamente necesario buscar soluciones a la situación de inseguridad jurídica de la ley y articular las fórmulas necesarias para garantizar la protección del patrimonio personal de los trabajadores ante eventuales situaciones de crisis en el futuro.

Por todo ello, entre otras modificaciones, uno de los principales objetivos de la nueva ley vasca, ha sido precisamente éste, el de tratar de alumbrar el camino a seguir en el futuro ante este tipo de situaciones ${ }^{26}$. Con ella se espera que el patrimonio de los socios quede blindado, o al menos se pueda garantizar su protección ${ }^{27}$.

\subsection{Régimen de imputación de pérdidas}

Como ocurría con la normativa estatal, la autonómica planteaba serias dudas sobre el límite de la responsabilidad de los socios. Justamente los que entraban en conflicto eran los artículos 56 y 69 de la antigua Ley 4/1993. En este caso, el artículo 56 , en su apartado primero, declaraba expresamente que "Los socios no responderán

23. UPAD del Juzgado de Primera Instancia e Instrucción nº 1 de Bergara, Sentencia nº. 70/2018, de 27 de julio, (Procedimiento ordinario 204/2017-C).

24. Sentencia de la Audiencia Provincial de Guipúzcoa, Sección segunda, de 1 de julio de 2019, sentencia nº. 515/2019 (Procedimiento ordinario. Ap. 2L 21356/2018-MR).

25. Vid. GOROSPE, P.: "El Parlamento vasco blinda el patrimonio de los cooperativistas", Diario El País, 20 diciembre, 2019.

26. Vid. la Exposición de motivos de la Ley de Cooperativas de Euskadi 11/2019: “(...) para la seguridad jurídica necesaria en la interpretación y aplicación de la norma cooperativa".

27. Vid. AZUMENDI, E.: "Euskadi protege el patrimonio de los cooperativistas tras la conmoción que supuso la caída del gigante Fagor”, elDiario.es, 24 diciembre, 2019. 
personalmente de las deudas sociales. Su responsabilidad por dichas deudas estará limitada a las aportaciones al capital social que hubieran suscrito".

Sin embargo, el artículo 69 de la Ley 4/1993, al regular la imputación de pérdidas, daba pie a la incertidumbre al establecer en su apartado 2.c), que "la cuantía no compensada con los fondos obligatorios y voluntarios se imputará a los socios en proporción a las operaciones, servicios o actividades realizadas por cada uno de ellos con la cooperativa"28.

El artículo 69 establecía que eran los estatutos los que debían de fijar los criterios para realizarla. Se podía optar por imputar las pérdidas a una cuenta especial, donde se compensarían con las ganancias futuras en el plazo de cinco años. En caso de que no se acudiera a esa cuenta especial las pérdidas debían de ser compensadas con los fondos de reserva voluntarios, si existiesen, y podrían imputarse todas las pérdidas. En caso de acudir a los fondos obligatorios, el porcentaje utilizable correspondería a la mitad de lo recaudado, (del excedente positivo) en los últimos cinco años. Una vez acudido a los fondos y no siendo suficiente su cuantía, existía la posibilidad de llevar a cabo la imputación a los socios teniendo en cuenta las operaciones, servicios o actividades de cada uno de ellos en la cooperativa.

Al efecto, la norma contenía dos modalidades. Era posible hacerlo directamente o acceder al importe aportado por el socio como capital social aplicando las correspondientes deducciones o echar mano de las inversiones realizadas por el socio dentro de la cooperativa. La segunda opción sería la de retener las cantidades que le correspondan recibir al socio por retornos durante los cinco ańos siguientes. En caso de haber seguido todo el proceso y no haber conseguido compensar todas las pérdidas, el socio debería de compensarlas en el plazo de un $\mathrm{mes}^{29}$.

28. Al respecto, vid. ALZOLA BERRIOZABALGOITIA, M.I.: "La responsabilidad de los socios de las cooperativas", legaltoday.com, 29 septiembre, 2009. Entiende que, a la hora de determinar el alcance de esta obligación, la asamblea general debe tener en cuenta lo dispuesto en la Ley, es decir, ello implica que la imputación debe realizarse "en proporción a las operaciones, servicios y actividades" realizadas por cada uno de los socios con la cooperativa.

29. Ley de Cooperativas de Euskadi 4/1993, artículo 69. Imputación de pérdidas.

1. Los Estatutos deberán fijar los criterios para la compensación de las pérdidas, siendo válido imputarlas a una cuenta especial para su amortización con cargo a futuros resultados positivos, dentro del plazo máximo de cinco años.

2. En la compensación de pérdidas la cooperativa habrá de sujetarse a las siguientes reglas:

a) A los fondos de reserva voluntarios, si existiesen, podrá imputarse la totalidad de las pérdidas.

b) Al Fondo de Reserva Obligatorio podrá imputarse como máximo el porcentaje medio de lo destinado a los fondos legalmente obligatorios en los últimos cinco años de excedentes positivos, o desde su constitución si ésta no fuera anterior a dichos cinco años. 
Atendiendo al tenor de la Ley 4/1993, extraemos la misma conclusión que con la ley estatal. Así, se deduce que la responsabilidad de los socios por las deudas de la sociedad cooperativa quedaba efectivamente limitada. En cambio, el panorama cambiaba ante las pérdidas de la sociedad. En esta situación, la jurisprudencia admitía la existencia de respaldo legal para poder involucrar al trabajador con todos sus bienes a fin de hacer frente a las pérdidas de la cooperativa y responder ante acreedores. Es por ello que los trabajadores exigían una protección superior a la que efectivamente se vio que tenían.

Éstos y otros episodios han impulsado que la concepción y la filosofía que acompañan a la cooperativa empiecen a buscar soluciones que se acercan a las de las sociedades capitalistas. Así, los socios trabajadores reclaman que su responsabilidad, bien por deudas o por pérdidas, sea limitada a la aportación realizada, como ocurre con los socios de las sociedades de capital. Este acercamiento no se considera extraño, habida cuenta de la necesidad de adaptar la normativa a la situación actual de la economía ${ }^{30}$. Aunque las cooperativas constituyan otro tipo jurídico de empresa, se entiende que en la práctica necesitan soluciones reales muy similares a las de las sociedades capitalistas, como puede ser necesidad de que la responsabilidad de los socios quede absolutamente limitada a la aportación realizada al capital social. Con la nueva regulación se han intentado aclarar los aspectos en los que surgen dudas de interpretación, generando en la práctica, como en el caso Fagor, serios riesgos para el patrimonio personal de los socios.

El artículo 73.1 de la Ley 11/2019 $9^{31}$, comienza diciendo lo mismo que el artículo 69 de la Ley 4/1993, indicando que "Los estatutos deberán fijar los criterios para la compensación de las pérdidas”, con sujeción a unas reglas casi idénticas a las anteriores. Prevé que las pérdidas podrán imputarse (a) a los fondos voluntarios, si existiesen, y (b) al fondo de reserva obligatorio. Para este supuesto añade que, si el fondo de reserva obligatorio superase el 50\% del importe del capital social, la cantidad excedente de dicho porcentaje podrá utilizarse para realizar la compensación de

c) La cuantía no compensada con los fondos obligatorios y voluntarios se imputará a los socios en proporción a las operaciones, servicios o actividades realizadas por cada uno de ellos con la cooperativa. Las pérdidas imputadas a cada socio se satisfarán de alguna de las formas siguientes:

1. Directamente o mediante deducciones en sus aportaciones al capital social o, en su caso, en cualquier inversión financiera del socio en la cooperativa que permita esta imputación, dentro del ejercicio siguiente a aquel en que se hubieran producido.

2. Con cargo a los retornos que puedan corresponder al socio en los cinco años siguientes. Si quedasen pérdidas sin compensar, éstas deberán ser satisfechas por el socio en el plazo máximo de un mes.

30. IRASTORZA MARTÍNEZ, I. \& LÓPEZ LOLO, R., op. cit.

31. Ley de Cooperativas de Euskadi 11/2019, artículo 73. Imputación de pérdidas. 
las pérdidas. (c) En caso de que estos fondos no fueran suficientes, se imputarán a los socios, en proporción a las operaciones, servicios o actividades realizadas por cada una de ellas con la cooperativa. Son unas reglas idénticas a las de la Ley 4/1993, con la salvedad de que se añade una regla más, prevista para las situaciones en que existan socios que no hayan realizado la aportación mínima para obtener la condición de socio. Para ese supuesto, se prevé que la imputación de las pérdidas sea proporcional a la actividad cooperativizada mínima obligatoria.

Una vez individualizada la imputación de pérdidas a los socios, la ley contempla dos posibilidades para satisfacerlas que deberá acordarse en la asamblea general. Ambas modalidades también son prácticamente idénticas a las de la Ley 4/1993. Una opción es que se satisfagan directamente o mediante deducciones en las aportaciones del socio al capital social o en cualquier inversión financiera que así lo permita, dentro del siguiente ejercicio económico. La otra es que se satisfagan con cargo a los retornos que puedan corresponder a la persona socia en los cinco años siguientes. Si quedasen pérdidas sin compensar, éstas deberán ser satisfechas por la persona socia en el plazo máximo de un año y no en el plazo de un mes como imponía el artículo 69 de la Ley 4/1993.

Asimismo, al lado de esta imputación de pérdidas estatutaria, el artículo 73.3 de la Ley 11/2019 sigue contemplando la posibilidad de imputarlas a una cuenta especial, pudiendo compensarlas con los excedentes de los próximos cinco años. $\mathrm{Al}$ respecto, la nueva ley especifica que, cuando esa compensación no fuera suficiente, se deberá de recurrir al orden establecido en el artículo 73.1: a) en primer lugar, a los fondos de reserva voluntarios; b) en segundo lugar, al fondo de reserva obligatorio y c) en tercer lugar, a los socios, en proporción a las operaciones o actividades realizadas con la cooperativa. En este último caso, exige que las cantidades sean satisfechas por el socio en el plazo de un año.

Como puede observarse, hasta aquí las novedades no son especialmente relevantes. Podría decirse, más bien, que la nueva ley introduce algunos ajustes al régimen de imputación de pérdidas heredado de la ley anterior. Sigue manteniendo las mismas dos modalidades, una imputación estatutaria, con cargo fundamentalmente a los fondos de reserva de la cooperativa y subsidiariamente a los socios, y la imputación a una cuenta especial con cargo a futuros excedentes.

La principal novedad de la Ley 11/2019 en el tema que nos ocupa se encuentra en el artículo 73.4, donde se prevé un tercer sistema, la ampliación de capital por parte de la cooperativa, a el fin de obtener los fondos necesarios para hacer frente a las pérdidas generadas. En este caso, las aportaciones corren a cargo de los socios, ya sea mediante nuevas aportaciones acordadas por la asamblea general o bien mediante nuevas aportaciones que sean necesarias para mantener la condición de socio en la 
cooperativa. Los socios deberán satisfacer dichas aportaciones, de lo contrario, deberán causar baja.

Así, el recurso a la ampliación de capital parece configurarse como una solución legal que opera in extremis, subsidiariamente, agotadas las otras dos vías, una vez transcurridos todos los plazos señalados para ellas. Ahora bien, del tenor literal del artículo 73.4 de la Ley 11/2019 parece desprenderse el carácter imperativo (no dispositivo) de la norma, que se aplicará si transcurridos esos plazos quedasen aún pérdidas sin compensar. El precepto, utilizando el modo verbal imperativo, ordena que dichas pérdidas "serán satisfechas mediante nuevas aportaciones", con independencia de que la cooperativa deba instar el concurso conforme a la ley concursal.

En definitiva, del análisis del régimen de imputación de pérdidas previsto en el artículo 73 de la Ley 11/2019, puede deducirse que contiene dos tipos de normas. Por un lado, contiene normas de carácter dispositivo, en virtud de las cuales se permite: (i) prever estatutariamente la compensación de pérdidas (apartados 1 y 2), o bien, (ii) imputar las pérdidas a una cuenta especial (apartado 3). Éstas son normas de derecho dispositivo que los sujetos destinatarios pueden excluir, es decir, tienen facultad para elegir una vía o la otra. Pero, por otro, también se contiene una norma de carácter imperativo (el apartado 4) de aplicación general, una norma que se aplicará necesariamente, en caso de que "queden aún pérdidas sin compensar". Para esos casos la ley impone la ampliación de capital de la cooperativa con cargo a nuevas aportaciones de los socios.

Parece que con esta fórmula innovadora el legislador busca, por una parte, que la cooperativa pueda sobrevivir y pueda revertir la mala situación económica inyectando dinero líquido que le sirva para compensar las pérdidas. Por otro, pretende asimismo aclarar las dudas legales derivadas de la normativa anterior, preservando a su vez el patrimonio personal de los socios, aunque sea de una manera sui generis. Mediante esta fórmula, en lugar de saldar las pérdidas, los socios realizan aportaciones a la sociedad, es decir, realizan inversiones, las cuales conforman activos de su patrimonio que, de este modo, no sufre menoscabo.

Sin embargo, es evidente que, aunque no se produzca una minoración del patrimonio del socio, sí queda afectado, pues su estructura no se mantiene inalterada. Debe matizarse que esos derechos patrimoniales derivados de las aportaciones a la sociedad no constituyen lo que la doctrina civilista denomina "derechos de disfrute", sino "derechos de obligación" ${ }^{2}$. Son derechos que le confieren a su titular la facultad 
de dirigirse contra otra persona (la sociedad cooperativa) y reclamar de ella un comportamiento ( $v g$. el derecho al retorno cooperativo o al reembolso, en caso de baja de la sociedad). Pero el titular no dispone de un poder directo e inmediato sobre ellos, como sucede con los derechos de disfrute.

Por todo ello, parece que el legislador ha pretendido proteger el patrimonio personal de los socios trabajadores, para casos como los de Fagor Electrodomesticos, pero no lo ha blindado absolutamente de todo efecto o circunstancia de forma que quede intacto. Parece que ha preferido buscar una solución innovadora conciliando esa protección del patrimonio personal con la responsabilidad de los socios por las pérdidas de la sociedad cooperativa.

\subsection{Baja del socio y deudas de la sociedad}

A este respecto, surge otra de las cuestiones que se plantean en torno al alcance de la responsabilidad limitada de los socios cooperativistas. ¿Qué ocurre cuando el socio se da de baja? ¿Sigue siendo responsable por las deudas contraídas por la sociedad? El artículo 15.4 LCoop estatal establece que el socio que haya causado baja en la sociedad seguirá siendo responsable de las deudas de la empresa durante los próximos cinco años. Con ello se entiende que sigue siendo responsable por las decisiones adoptadas durante su permanencia en la cooperativa ${ }^{33}$.

Ahora bien, esta responsabilidad, tal y como ocurre con los socios de la cooperativa, será limitada, en este caso, hasta el importe reembolsado de sus aportaciones al capital social. Es decir, el socio responderá con el importe que hubiera aportado para adquirir la condición de socio de la cooperativa.

El socio que causa baja cuenta con el derecho de reembolso de la aportación realizada $^{34}$. Aunque en opinión de algunos autores no existe el derecho de reembolso, sino el derecho de liquidación de la aportación realizada ${ }^{35}$. Esto es, la devolución que realiza la cooperativa al socio que efectuó la contribución al capital social ha de ser

33. LCoop artículo 15.4: "No obstante, el socio que cause baja en la cooperativa responderá personalmente por las deudas sociales, previa exclusión del haber social, durante cinco años desde la pérdida de su condición de socio, por las obligaciones contraídas por la cooperativa con anterioridad a su baja, hasta el importe reembolsado de sus aportaciones al capital social".

34. LCoop artículo 45.1.a): "El capital social estará constituido por las aportaciones obligatorias y voluntarias de los socios, que podrán ser: a) aportaciones con derecho de reembolso en caso de baja”.

35. Vid. VARGAS VASSEROT, C.: "El derecho de reembolso del socio en caso de baja y el concurso de las sociedades cooperativas", CIRIEC-España, Revista Jurídica de economía social y cooperativa, $\mathrm{n}^{\circ} 21$, diciembre, 2010, pp. 37-58. 
puesto al día conforme a los excedentes repartibles y las pérdidas imputables generadas por la sociedad teniendo en cuenta el resultado de su actividad ${ }^{36}$. Sin embargo, sucede que dicha devolución normalmente no suele ser inmediata, por lo que, durante los cinco ańos siguientes a la baja el ex-socio seguirá siendo responsable de las deudas generadas $\mathrm{y}$, a falta de devolución, responderá con la aportación realizada ${ }^{37}$. Sin embargo, algunos autores estiman oportuno que la devolución de las aportaciones sea inmediata ${ }^{38}$.

En este punto, se plantea la interrogante de ante qué deudas debe de responder el socio. Esto es, esas deudas ¿deben haber sido generadas durante el período en que estuvo activo en la empresa y tuvo conocimiento de ellas? ¿puede tratarse de deudas generadas cuando ya no se encontraba en la cooperativa y no tenía conocimiento?

Para algunos expertos la posibilidad de la dualidad de la responsabilidad sería una opción correcta $^{39}$. Por el contrario, la LCoop exige que la responsabilidad debe de ser generada expresamente cuando el socio haya tenido la condición de socio de la empresa, por actos anteriores a la baja, pero no por actos llevados a cabo con posterioridad ${ }^{40}$, al igual que ocurre con el reparto de beneficios. El socio trabajador recibe las ganancias cuando se encuentra activamente en la empresa ${ }^{41}$, pero una vez que haya causado baja, no le asiste el derecho a percibir los importes de las ganancias repartidas. Por tanto, la lógica impone que la misma regla sea aplicada para el caso de las deudas.

Sin embargo, la situación en Euskadi es algo distinta. La Ley 4/1993 se limitaba a disponer que el socio no respondía personalmente por las deudas sociales y que, tras causar baja, una vez establecido el importe a reembolsar, -habrá que tener en cuenta

36. Vid. VIGUERA REVUELTA, R.: El Derecho de reembolso en las sociedades cooperativas, Tirant lo Blanch, València, 2015, p. 115.

37. Vid. BATALlER GRAU, J. (dir.): Pérdidas, disolución y concurso en sociedades cooperativas, Marcial Pons, Ediciones Jurídicas y Sociales, Madrid, 2012, pp. 133 y ss.

38. Vid. NAGORE APARICIO, I.: "Reflexiones sobre diversas cuestiones cooperativas (análisis principal bajo Ley Vasca)”, Deusto Estudios Cooperativos, no 13, julio, 2019, p. 46.

39. Vid. PAZ CANALEJO, N., op. cit., pp. 196-197.

40. LCoop artículo 15.4.: "por las obligaciones contraídas por la cooperativa con anterioridad a su baja”.

41. LCoop artículo 48.2: "La remuneración de las aportaciones al capital social estará condicionada a la existencia en el ejercicio económico de resultados positivos previos a su reparto, limitándose el importe máximo de las retribuciones al citado resultado positivo y, en ningún caso, excederá en más de seis puntos del interés legal del dinero". 
si la baja se ha considerado justificada o no justificada ${ }^{42}$-, tampoco sería responsable de las deudas contraídas por la cooperativa anteriores a su baja ${ }^{43}$. Por consiguiente, en el caso de baja del socio, la responsabilidad quedaba claramente limitada ${ }^{44}$.

Con la Ley 11/2019 se ratifica que es la cooperativa la que hará frente a las deudas sociales con su patrimonio presente y futuro, exceptuando las contribuciones para la educación y promoción cooperativa y otros fines de interés público ${ }^{45}$. Así se admite que los socios no responderán personalmente con su patrimonio ante las deudas y que su responsabilidad queda limitada a las aportaciones realizadas ${ }^{46}$.

En, caso de baja del socio, una vez se haya acordado el importe a reembolsar, éste no responderá por las deudas contraídas por la sociedad anteriores a su marcha. Ésa es la regla general que contiene al artículo 59.3 de la Ley 11/2019 y se reconoce, asimismo, la posibilidad de solicitar el reembolso correspondiente de las reservas repartibles ${ }^{47}$.

No obstante, el artículo 59.4 de la Ley 11/2019 recoge una matización a esa regla general que constituye un cambio normativo relevante respecto al régimen de la baja del socio $^{48}$. Dispone que el socio que haya causado baja será responsable del cumpli-

42. Vid. GONDRA ELGUEZABAL, G. \& BILBAO ZORROZUA, A.: "Alcance del régimen de responsabilidad de los socios en las cooperativas de viviendas en Euskadi", Gizarte Ekonomiaren Euskal Aldizkaria/Revista Vasca de Economía Social, no 16, 2019, pp. 149-166.

43. Ley 4/1993, artículo 56.2: "Una vez fijado el importe de las aportaciones a reembolsar, los socios que causen baja, no tendrán responsabilidad alguna por las deudas que hubiese contraído la cooperativa con anterioridad a su baja”.

44. Vid. OTXOA-ERRARTE GOICOECHEA, R.: "A propósito del Laudo del Servicio Vasco de Arbitraje Cooperativo n. ${ }^{\circ}$ 7/2018, del 20 de julio de 2018. Doble contabilidad y asunción de pérdidas", Gizarte Ekonomiaren Euskal Aldizkaria/Revista Vasca de Economía Social, no 16, 2019, p. 183.

45. Ley 11/2019, artículo 59. 1: "La cooperativa responderá por las deudas sociales con todo su patrimonio presente y futuro, excepto el correspondiente a la contribución para la educación y promoción cooperativa y otros fines de interés público, que solo responderá de las obligaciones contraídas para el cumplimiento de sus fines".

46. Ley 11/2019, artículo 59.2: "Las personas socias no responderán personalmente de las deudas sociales. Su responsabilidad por dichas deudas estará limitada a las aportaciones al capital social que hubieran suscrito".

47. Ley 11/2019, artículo 59.3: "Una vez fijado el importe de las aportaciones a reembolsar, las personas socias que causen baja no tendrán responsabilidad alguna por las deudas que hubiese contraído la cooperativa con anterioridad a su baja". Asimismo, el artículo 63.4. "Si la asamblea general acuerda devengar un interés a las aportaciones o destinar excedentes disponibles a retornos o a reservas repartibles, las aportaciones previstas en el artículo 60.1.b cuyo reembolso hubiera sido rehusado por la cooperativa y cuyos titulares hubieran causado baja, tendrán una remuneración preferente que se establecerá en los estatutos sociales".

48. Ley 11/2019, artículo 59.4: "Las personas socias que hubieran, expresa y específicamente, suscrito contratos o asumido obligaciones con la sociedad cooperativa y que, por su naturaleza, no se extinguen con la pérdida de la condición de persona socia responderán de su cumplimiento aún después de causar baja”. 
miento de aquellas obligaciones que hubiera asumido o contratos que hubiera suscrito expresa y específicamente con la sociedad cooperativa y que, por su naturaleza, no se extingan a pesar de causar baja. Es decir, seguirá siendo responsable si, debido a su naturaleza, el contrato sigue activo, aunque el socio se haya dado de baja. En ese caso el socio deberá de responder por su cumplimiento. En caso contrario, la cooperativa podrá retener el importe que le corresponda recibir al socio que ha causado baja ${ }^{49}$.

En definitiva, el legislador pretende cubrir una situación donde el socio que haya causado baja no se desvincule fácilmente de las obligaciones asumidas, y así, lograr que la pervivencia de la cooperativa se pueda prolongar en el tiempo con la implicación de todos los actores protagonistas.

\section{Conclusiones}

Las crisis económicas sacan a relucir las debilidades del sistema económico y del sistema jurídico, como ocurrió con el régimen de la responsabilidad de los socios de la cooperativa en el contexto de la crisis de 2007.

En el marco de la ley de cooperativas estatal, que opera como referencia básica rodeada de leyes autonómicas, se plantean dudas acerca del alcance limitado de la responsabilidad de los socios de las cooperativas. Si bien, el artículo 15.3 LCoop dispone que la responsabilidad del socio por las deudas sociales estará limitada a las aportaciones al capital social que hubiera suscrito, el artículo 59 LCoop, al regular la imputación de pérdidas de la sociedad cooperativa, contempla la posibilidad de imputarlas a los socios.

Un entendimiento sistemático, del conjunto de la ley, nos conduce a la conclusión de que la intención reguladora del legislador y, por tanto, la finalidad de la norma es la de distinguir dos conceptos, deuda y pérdida, y dos supuestos de responsabilidad asociados a ellos. Al respecto, la responsabilidad limitada de los socios por las deudas de la cooperativa no ofrece dudas, pero no sucede así en el caso de la responsabilidad por las pérdidas, por lo que persiste la ambigüedad de la norma.

La misma situación se repite en el País Vasco, cuya economía se caracteriza por la fuerte presencia de sociedades cooperativas y donde la crisis del 2007 dejó en una situación económica complicada a muchas de ellas, como fue el caso de Fagor Electrodomésticos. Con la ley 4/1993 de cooperativas de Euskadi, los trabajadores no 
consiguieron blindar su patrimonio personal ante las consecuencias derivadas de la insolvencia de la empresa.

Éste y otros hechos dieron motivo a una revisión de la situación normativa que culminó con la aprobación de la nueva Ley 11/2019 de cooperativas que entró en vigor en enero de 2020. Una vez más, queda claro que los socios son responsables de las deudas sociales generadas en la cooperativa, hasta el límite de la aportación realizada para adquirir la condición de socio. Aunque también hay que señalar que la nueva ley prevé prorrogar la responsabilidad por deudas de los socios, aún después de causar baja, en caso de que hubieran suscrito contratos o asumido obligaciones con la sociedad cooperativa que, por su naturaleza, no se extinguen con la pérdida de la condición de socio.

Por lo que respecta a la responsabilidad de los socios por las pérdidas de la sociedad cooperativa, el artículo 73 de la Ley 11/2019 sigue manteniendo las dos modalidades opcionales de prever estatutariamente la compensación de pérdidas, o bien, imputarlas a una cuenta especial. Pero añade una nueva previsión imponiendo la ampliación de capital de la cooperativa con cargo a nuevas aportaciones de los socios para el caso de que queden aún pérdidas sin compensar.

Parece que el legislador ha querido aclarar las dudas legales derivadas de la normativa anterior, con una fórmula que pone el foco en revertir la mala situación económica de la cooperativa y asegurar su pervivencia, preservando a su vez el patrimonio personal de los socios de una manera sui generis. Prevé que, en lugar de contribuir a abonar las pérdidas, los socios inyectan fondos a la cooperativa realizando aportaciones al capital social, por lo que su patrimonio no se ve menguado, aunque obviamente su estructura queda alterada. De este modo, el legislador llega a proteger el patrimonio personal de los socios, aunque no a blindarlo absolutamente de todo sacrificio, por lo que parece que ha buscado una solución innovadora que concilia esa protección con la responsabilidad de los socios por las pérdidas de la sociedad cooperativa.

En este contexto de crisis generada por la COVID 19, habrá que ver las repercusiones de su impacto en lo económico y en lo social y las carencias normativas que la nueva realidad pueda dejar en evidencia. 


\section{Bibliografía}

ALZOLA BERRIOZABALGOITIA, M.I.: El régimen jurídico de los fondos de reserva voluntarios de las sociedades cooperativas [Tesis doctoral, Mondragón Unibertsitatea], Mondragón, 2009.

ALZOLA BERRIOZABALGOITIA, M.I.: "La responsabilidad de los socios de las cooperativas", legaltoday.com, 29 septiembre, 2009. Recuperado 1 de junio de 2020, de: http://www.legaltoday.com/practica-juridica/mercantil/cooperativas/ la-responsabilidad-de-los-socios-de-las-sociedades-cooperativas

ALZOLA BERRIOZABALGOITIA, M.I. \& VILLAFÁŃEZ PÉREZ, I.: "Los recursos financieros en la legislación cooperativa y sus peculiaridades en las cooperativas de trabajo asociado". En: Cooperativa de trabajo asociado y estatuto jurídico de sus socios trabajadores (dir. FAJARDO GARCÍA, I.G. \& coord. SENENT VIDAL, M.J.), Tirant lo Blanch, València, 2016, pp. 481-512.

ARCO ÁLVAREZ, J.L.: Teoría y práctica de las sociedades cooperativas. Temas de estudio de los Cursos I, II y III, Universitat Politècnica de València (UPV), València, 1974.

ARRIETA IDIAKEZ, F.J.: La baja como causa de finalización de la relación societaria entre la persona socia y la sociedad cooperativa, Dykinson, Madrid, 2019.

ATIENZA MAZIAS, E., MERINO MAR, J.A. \& GUILLERMO RUIZ DE HUYDOBRO Y DE LAS MUNEECAS, E.: "Derecho de las Sociedades Cooperativas en Euskadi: Un análisis sobre su estructura orgánica y política cooperativa de los últimos ejercicios", Boletín de la Asociación Internacional de Derecho Cooperativo (BAIDC), no 38, 2004, pp. 105-191.

DOI: https://doi.org/10.18543/baidc-38-2004pp105-191

AZUMENDI, E.: "Euskadi protege el patrimonio de los cooperativistas tras la conmoción que supuso la caída del gigante Fagor", elDiario.es, 24 diciembre, 2019. Recuperado el 19 de mayo de 2020, de https://www.eldiario.es/euskadi/euskadi/euskadi-patrimonio-cooperativistas-conmocion-fagor_1_1173528.html

BAKAIKOA AZURMENDI, B.: "El cooperativismo vasco. El ejemplo de la Mondragón Corporación Cooperativa”. En: El cooperativismo Vasco y el año 2000, Marcial Pons, Ediciones Jurídicas y Sociales \& Gezki, Madrid, 1995, pp. 29-47. 
BAKAIKOA AZURMENDI, B., ETXEZARRETA ETXARRI, E. \& MORANDEIRA ARCA, J.: "Regeneración de empresas de la economía social: un reto en el País Vasco", REVESCO, Revista de Estudios Cooperativos, no 112, noviembre, 2013, pp. 151-175. DOI: https://doi.org/10.5209/rev_REVE.2013.v112.43064

BATALLER GRAU, J. (dir.): Pérdidas, disolución y concurso en sociedades cooperativas, Marcial Pons, Ediciones Jurídicas y Sociales, Madrid, 2012.

BELTRÁN SÁNCHEZ, E.M.: "Reforma de la Ley de cooperativas". En: Comentario de la Ley Concursal (dirs. ROJO FERNÁNDEZ-RÍO, A., BELTRÁN SÁNCHEZ, E.M. \& coord. CAMPUZANO LAGUILLO, A.B.), Civitas, Madrid, 2006, pp. 3267-3268.

DÍEZ PICAZO, L.: Fundamentos del Derecho civil patrimonial I. Introducción teoría del contrato, Civitas, Madrid, 1996.

ELENA DÍAZ, F.: "Ideas sobre una posible interpretación de las pérdidas del pasivo según la vigente ley”, Estudios cooperativos, no 36-38, 1975-1976. pp. 185-224.

EMBID IRUJO, J.M.: "El relieve del derecho de sociedades para la ordenación jurídica de las empresas de economía social”, Cuadernos de Derecho y Comercio, no. 71, 2019, pp. 15-48.

FAJARDO GARCÍA, I.G.: "La gestión económica de las cooperativas: responsabilidad de los socios". En: Confederación de cooperativas de la comunidad Valenciana, Capitulo V, Editorial Tecnos, Madrid, 1997, pp. 197-245.

GADEA SOLER, E., SACRISTÁN BERGIA, F. \& VARGAS VASSEROT, C.: Régimen jurídico de la sociedad cooperativa del siglo XXI. Realidad actual y propuestas de reforma, Dykinson, Madrid, 2009.

GONDRA ELGUEZABAL, G. \& BILBAO ZORROZUA, A.: "Alcance del régimen de responsabilidad de los socios en las cooperativas de viviendas en Euskadi”, Gizarte Ekonomiaren Euskal Aldizkaria/Revista Vasca de Economia Social, no 16, 2019, pp. 149-166. DOI: https://doi.org/10.1387/reves.21217.

GOROSPE, P.: "El Parlamento vasco blinda el patrimonio de los cooperativistas", Diario El Pais, 20 diciembre, 2019. Recuperado el 20 de mayo de 2020, de https://elpais.com/economia/2019/12/20/actualidad/1576835081_858504. html

GRIMALDOS GARCÍA, M.I.: "El concurso de la cooperativa y su repercusión en el patrimonio personal de los socios", Revista de Derecho Concursal y Paraconcursal, $\mathrm{n}^{\circ}$. 11, 2009, pp. 323-343.

IRASTORZA MARTÍNEZ, I. \& LÓPEZ LOLO, R.: “Acercamiento del régimen de las sociedades cooperativas al de las sociedades de capital. ¿Un cambio necesario para el futuro del modelo cooperativo?”, Diario la Ley, no. 9582, 2020. 
LARRAÑAGA, J.: Análisis de la Legislación Vasca sobre cooperativas, Caja Laboral Popular, Mondragón, 1985.

MATEO BLANCO, J.: El retorno cooperativo, Caja Rural de Zaragoza, Zaragoza, 2003.

NAGORE APARICIO, I.: "Reflexiones sobre diversas cuestiones cooperativas (análisis principal bajo Ley Vasca)", Deusto Estudios Cooperativos, no 13, julio, 2019, pp. 29-67.

ORTEGA I.: Aportación de las Cooperativas al resto del tejido vasco, Mondragon Unibertsitatea, Mondragón, 2018.

OTXOA-ERRARTE GOICOECHEA, R.: "A propósito del Laudo del Servicio Vasco de Arbitraje Cooperativo n. ${ }^{\circ}$ 7/2018, del 20 de julio de 2018. Doble contabilidad y asunción de pérdidas", Gizarte Ekonomiaren Euskal Aldizkaria/Revista Vasca de Economía Social, no 16, 2019, pp. 169-185.

DOI: https://doi.org/10.1387/reves.21220.

PAZ CANALEJO, N.: El nuevo derecho cooperativo español, Digesa, Madrid, 1979. SOTO GORROTXATEGI, A., \& MORANDEIRA ARCA, J.: Informe de situación de la Economía Social Vasca año 2016, Observatorio Vasco de Economía Social I Gizarte Ekonomiako Euskal Behatokia (OVES/GEEB) \& Gezki, Donostia, 2016.

VARGAS VASSEROT, C.: "El derecho de reembolso del socio en caso de baja y el concurso de las sociedades cooperativas", CIRIEC-España, Revista Jurídica de Economía Social y Cooperativa, no 21, diciembre, 2010, pp. 37-58.

VICENT CHULIÁ, F.: Ley general de cooperativas, Tomo XX de los Comentarios al Código de comercio y Legislación mercantil Especial, vol. 3, Madrid, 1994.

VICENT CHULIÁ, F.: Introducción al derecho mercantil, vol. 1, Tirant lo Blanch, València, 2012.

VIGUERA REVUELTA, R.: El Derecho de reembolso en las sociedades cooperativas, Tirant lo Blanch, València, 2015.

VILLA GÓMEZ, A.: Comentarios a la ley de cooperativas 27/1999 de 16 de julio, vol. I, Madrid, 2001.

VITERI ZUBIA, I.: "La responsabilidad del socio cooperativista por las pérdidas sociales", CIRIEC-España, Revista Jurídica de Economía Social y Cooperativa, ${ }^{\circ}$ 28, julio, 2016, pp. 209-245. 